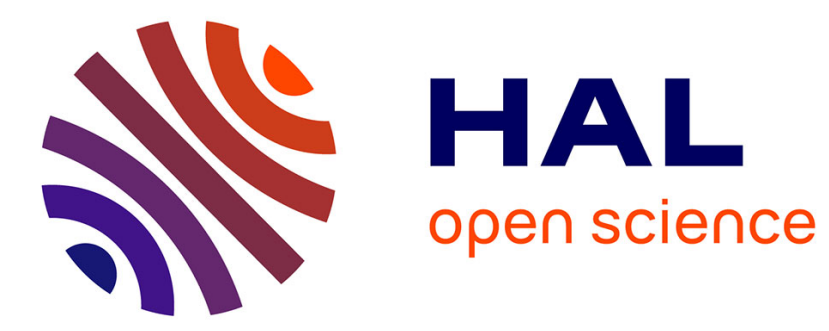

\title{
Femtosecond reactivity of electron in aqueous solutions
} Yann Gauduel, A. Migus, J.P. Chambaret, A. Antonetti

\section{To cite this version:}

Yann Gauduel, A. Migus, J.P. Chambaret, A. Antonetti. Femtosecond reactivity of electron in aqueous solutions. Revue de Physique Appliquée, 1987, 22 (12), pp.1755-1759. 10.1051/rphysap:0198700220120175500 . jpa-00245736

\section{HAL Id: jpa-00245736 https://hal.science/jpa-00245736}

Submitted on 1 Jan 1987

HAL is a multi-disciplinary open access archive for the deposit and dissemination of scientific research documents, whether they are published or not. The documents may come from teaching and research institutions in France or abroad, or from public or private research centers.
L'archive ouverte pluridisciplinaire HAL, est destinée au dépôt et à la diffusion de documents scientifiques de niveau recherche, publiés ou non, émanant des établissements d'enseignement et de recherche français ou étrangers, des laboratoires publics ou privés. 


\title{
Femtosecond reactivity of electron in aqueous solutions
}

\author{
Y. Gauduel, A. Migus, J. P. Chambaret and A. Antonetti \\ Laboratoire d'Optique Appliquée, INSERM U275, ENS Techniques Avancées, Ecole Polytechnique, \\ 91120 Palaiseau, France
}

(Reçu le 12 juin 1987, accepté le 9 juillet 1987)

\begin{abstract}
Résumé. - Les techniques optiques femtosecondes qui permettent d'engendrer des impulsions intenses du proche UV au proche infrarouge sont utilisées pour étudier des réactions physicochimiques et photochimiques ultra-rapides dans des solutions aqueuses. Des résultats de l'étude dynamique de la solvatation d'un électron dans l'eau et une solution saline concentrée seront discutés.
\end{abstract}

\begin{abstract}
Femtosecond optical techniques allowing the generation of intense optical pulses from the near ultraviolet to the near infrared are used in the monitoring of ultrafast photophysical and photochemical reactions in aqueous solutions. Selected examples for dynamics of electron solvation in water and aqueous solution of ferrocyanide are discussed.
\end{abstract}

\section{Introduction.}

Since its discovery more than twenty five years ago the hydrated electron continues to be of considerable interest and a matter of debate and controversy [1]. It is a species of prime importance in chemistry, biology and even technology because the energetics and the time dependence of the electron-medium interaction play an important role in the formation of this radical. It is also a theoretical challenge since the solvated electron presents the particular property that its structure is totally determined by the solvent. There has been continuing discussion over the relative role and chronological importance in the long-range and short-range electron-medium interactions in the sequence of electron trapping and solvation $[2,4]$.

Following a dielectric continuum picture of the solvent it has been suggested that the transition toward solvation in polar liquids is initiated by longrange dipolar interactions followed by self-trapping of the electron. This latter scheme has been favoured in liquid alcohols since a precursor of the solvated electron has been identified through an infrared absorption which extends to the visible and which decays within ten to a few hundred picoseconds, depending on the alcohol chain length.This has been interpreted as an electron localized in a shallow trap or a fluctuation of the surface potential, evolving from there toward the solvated state via a configura- tional relaxation of the medium in the electronic field of the charge. Until quite recently, only the fully relaxed solvated electron could be observed in liquid water, raising the assumption of direct localization of excess electron in pre-existing trapping sites [5].

Important theoretical breakthroughs have been realized recently by using computer simulations. Several groups have thus deduced some (conflicting) information on the structural aspect of the hydrated electron by employing path integral techniques while a molecular dynamic simulation of water has identified favorable sites for the initial trapping of electrons [6-9]. This fast evolution of computer simulations lacked however experimental grounds.

Employing femtosecond spectroscopy technique we have found unique information on the dynamics of both trapping and solvation of electron in aqueous solutions. While an unambiguous identification of the transient states cannot be reached directly by spectroscopy alone, these dynamical data demonstrate that electron solvation in water proceeds through at least one intermediate state whose lifetime is in the femtosecond regime.

In the following we will present the experimental scheme and results in the two cases of direct photoionization of a pure water molecule and photolysis of an aqueous solution of ferrocyanide ion. We will discuss our data in the framework exposed above. 


\section{Methods.}

The recent advances in ultrashort laser pulse generation permit the transfer to the picosecond and femtosecond time scale of most of the already developed optical methods making possible an accurate determination of the kinetics of electron solvation in polar medium. In such liquids, the electron solvation can intervene when an excess electron is ejected from a solvent or solute molecule. Spectral and kinetic investigations of the reactivity of an electron in polar media, have been carried out using a femtosecond pump-probe method $[10,11]$. The experimental apparatus (Fig. 1) is based on a laser amplifier system producing high peak power tunable pulses of typically $100 \mathrm{fs}$ duration. After passing through a variable delay line driven by a stepping motor, one beam is focused into a KDP crystal to produce up to $20 \mu \mathrm{J}, 100 \mathrm{fs}$ at $310 \mathrm{~nm}$ (Fig. 1). The UV beam (pump or excitation beam) is then focused on a $300 \mu \mathrm{m}$ diameter spot in the $2 \mathrm{~mm}$ long sample and initiates the ionization process. The other half of the $620 \mathrm{~nm}$ beam is focused into a $2 \mathrm{~cm}$ water cell and converted to a white light continuum. A part of the beam is selected with colored filters and split into two beams : one beam probes collinearly the excited part of the sample (probe or test beam) while the other (reference beam) misses the cuvette.

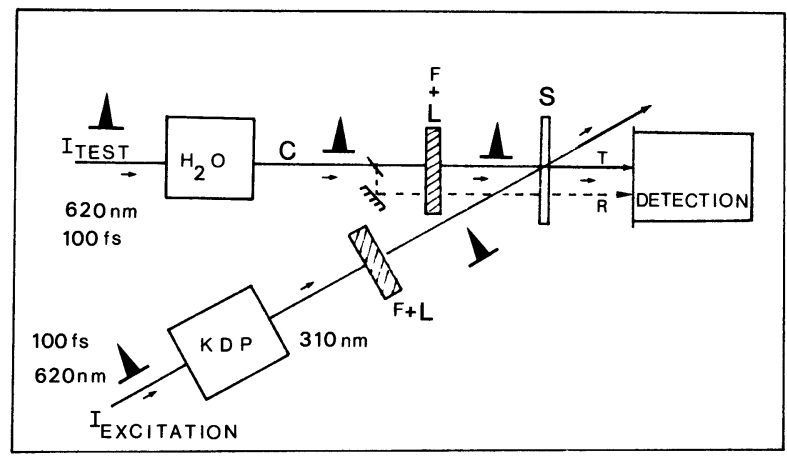

Fig. 1. - Experimental arrangement for femtosecond absorption studies. Input beam at $620 \mathrm{~nm}$ is split in two parts : one is the test beam and the other the excitation beam. The test beam, a weak white light generated in a water cell is split in two parts : one probes the region of the sample and the other is used as a reference. F : filter, $\mathrm{L}$ : lens, $\mathrm{C}$ : continuum, $\mathrm{T}$ : test beam, $\mathrm{R}$ : reference beam, $\mathrm{S}$ : sample.

The two signals corresponding to the probe and reference pulse energy at a given wavelength are sent through an electronic chain (pulse shaping, sampling holder, digitizer) to a computer. This laser and amplifier set up coupled to this absorption spectroscopy detection scheme allowed us to measure changes in the optical density $A<10^{-3}$ with femtosecond accuracy.
One key point in this experiment is the determination for each probe wavelength of both the instrumental instantaneous response and the zero time delay (coincidence) between the pump and the probe pulses. This has been done by observation of a very weak instantaneous induced absorption in a pure $n$-heptane solution excited at $310 \mathrm{~nm}$ in the same condition as water [11].

\section{Results.}

3.1 Electron SOlVATION IN PURE WATER. - Up to now the great majority of electron solution studies in pure water has been realized by injecting electron using pulse radiolysis methods, providing at best a $10 \mathrm{ps}$ accuracy. On the other hand, direct photoionization of water molecules by visible and near UV light does not seem possible since water is transparent in the UV spectral region up to $190 \mathrm{~nm}$. However in our case of ultrashort pulse, light can be absorbed through a non linear process, namely two photon absorption [12]. Indeed the two photon absorption coefficient of pure water at $310 \mathrm{~nm}$ has been estimated to be $4 \times 10^{-13} \mathrm{~m} / \mathrm{W}$ yielding non negligible absorption when dealing with multigigawatt peak power pulses. In this case our $8 \mathrm{eV}$ twophoton excitation is above the ionization threshold for liquid water, estimated to be around $6.5 \mathrm{eV}$ (Fig. 2). So, each 100 femtosecond pulse produces a homogeneous solvated electron concentration of about $5 \mu \mathrm{M}$ and furthermore permits the dynamics of electron solvation to be resolved with femtosecond

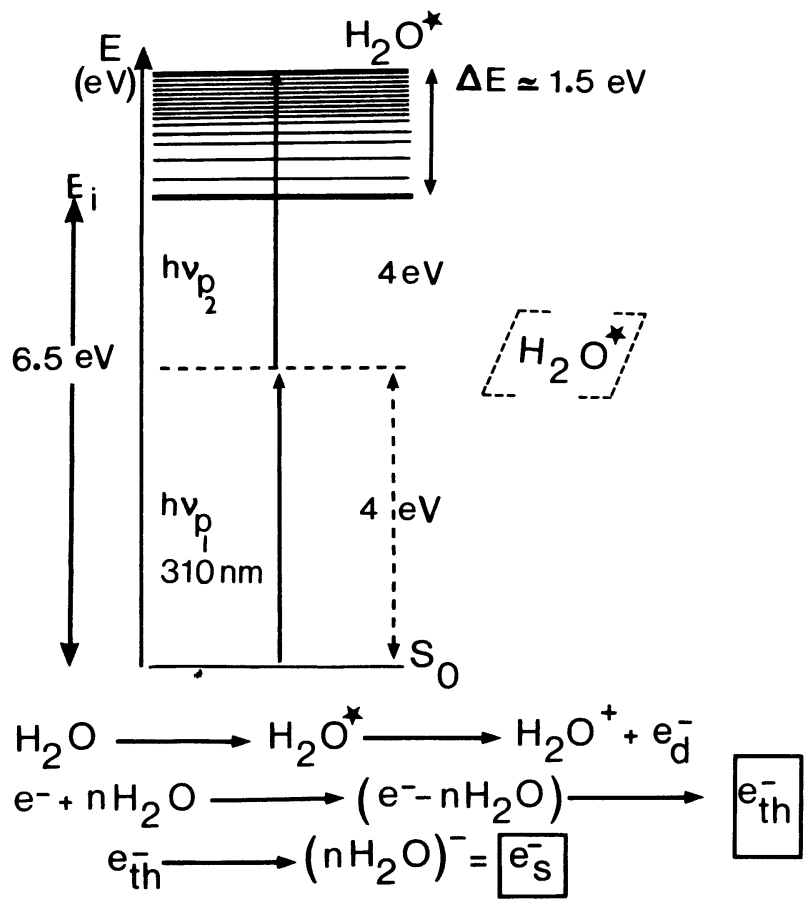

Fig. 2. - Scheme for femtosecond photoionization of liquid water at $294 \mathrm{~K}$ using high power UV pulses $\left(\lambda_{\mathrm{c}}=310 \mathrm{~nm}\right.$ i.e. $\left.E=4 \mathrm{eV}\right)$. 
accuracy, starting from initially quasi free state up to full solvation.

Induced absorption kinetics measured over the whole visible spectrum and in the near infrared up to $1250 \mathrm{~nm}$ have been used to reconstitute a transient spectrum. The high energy tail of an infrared band extending above $1250 \mathrm{~nm}$ appears within the excitation and is fully developed after a delay of $0.2 \mathrm{ps}$ (Fig. 3). This IR band then decays concurrently with the rise of an absorption band which peaks around $720 \mathrm{~nm}$ and which coincides with the known solvated electron one. The kinetics at 1250 and $720 \mathrm{~nm}$ reflect the evolution of the infrared and solvated species respectively (Fig. 3). The infrared band appears with a time constant of $110 \pm 20 \mathrm{fs}$ and relaxes following a first order kinetics with a time constant of $240 \pm 20$ fs towards the solvated species.
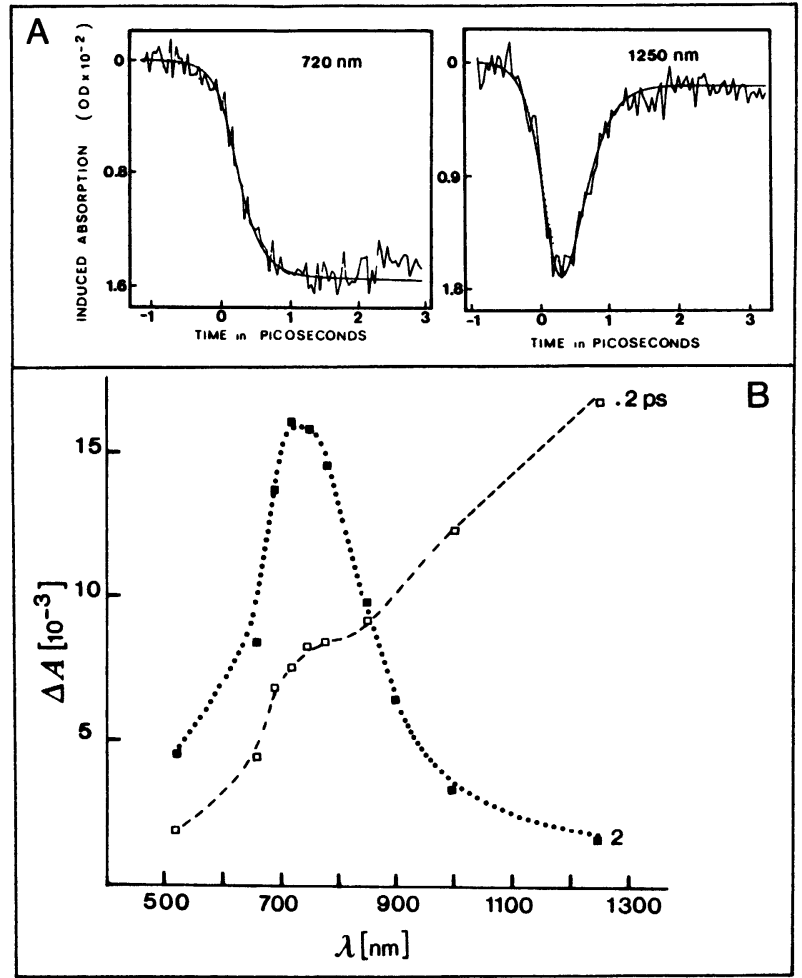

Fig. 3. - Absorption spectra of the electron at $200 \mathrm{fs}$ and 2 ps after femtosecond photoionization of liquid water at $294 \mathrm{~K}$. Insert : time-resolved data obtained at $1250 \mathrm{~nm}$ and $720 \mathrm{~nm}$ following femtosecond photoionization of liquid water. The smooth lines represent the computed best fits assuming an appearance time of $110 \mathrm{fs}$ for the infrared species $(1250 \mathrm{~nm})$ and its relaxation towards the solvated state in $240 \mathrm{fs}$.

These data are the first evidence of the existence of at least one transient intermediate of electron solvation in pure water. What is the nature of this state ? If we follow the recent work of P. Rossky's group [9] possible initial preexisting trapping sites are arising from statistical fluctuations and molecular clustering. They find a distribution of such sites monotonically in energy down to $-1.4 \mathrm{eV}$, which is compatible with our IR data. We have proposed that this precursor is a state (presolvated state) where the electron is still spatially extended [13, 14]. Is it already a thermalized state ? This would imply that the excess kinetics energy ( $1.5 \mathrm{eV}$ at maximum) has been transferred to the water molecules in less than the risetime $T_{1}$ of the IR spectrum, i.e. $100 \mathrm{fs}$. Such fast thermalization is quite possible if we take into account the existing numerous modes of vibration of the solvent. These dynamics can in fact be compared to the dynamics of electron trapping in the shallow part of the exponential band as tail states in the amorphous materials process which has been demonstrated to occur in the femtosecond regime [15]. In this case the local temperature rise can be estimated to be negligible since the solvated electron spectrum taken only a fraction of picosecond after creation, and which is sensitive to temperature, is exactly as expected for $\theta=294 \mathrm{~K}$.

Turning toward the transition to the fully solvated state, it has been suggested as mentioned earlier that once the electron gets localized, it evolves toward the solvated state via a configurational relaxation of the medium in the electronic field of the charge. Theories developed in this framework predict that this relaxation should occur through a continuous shift of the electron absorption with a characteristic time of the order of $T_{\mathrm{L}}=0.240 \mathrm{ps}$ where $T_{\mathrm{L}}=$ $T_{\mathrm{D}} \varepsilon_{\infty} / \varepsilon_{0}$ with $T_{\mathrm{L}}$ the longitudinal relaxation time, $T_{\mathrm{D}}$ the Debye relaxation time, $\varepsilon_{\infty}\left(\varepsilon_{0}\right)$ being the high/(low) frequency limit of the dielectric constant [16]. Indeed we observe a decay of the IR species with a time constant $T_{2}$ close to $T_{\mathrm{L}}$ and a concomitant rise of the known solvated electron spectrum. A picture of separate transient electronic states of electron would then be consistent with our data,

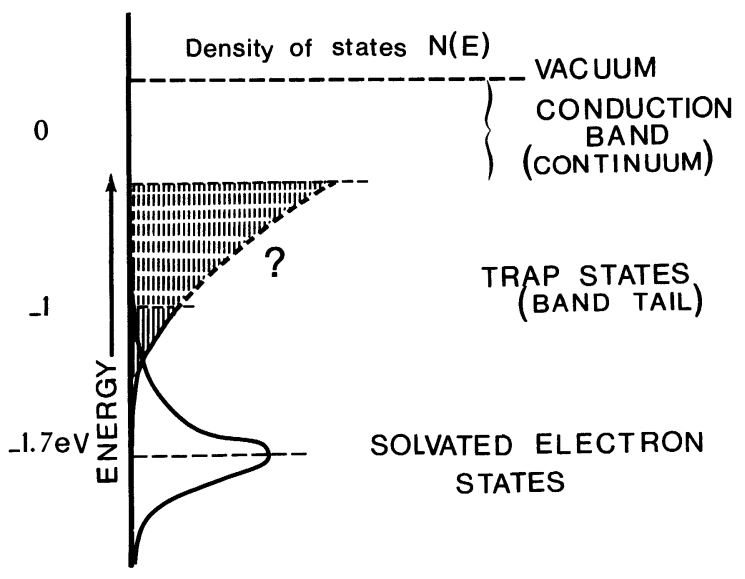

Fig. 4. - Schematic representation of the energy diagram and density states for presolvated and solvated electron in liquid water. 
although we cannot completely rule out a dielectric relaxation of the medium. We may for instance assume a continuous shift of the energy of the electron associated with a decrease of Franck-Condon factors while the solvent has not reached its equilibrium. In this case the absorption of light by the electron would be negligible in the dynamic phase.

We may also notice that in our case the solvent is modified because we do not inject electrons but take them from a water molecule and create consequently a cation $\left(\mathrm{H}_{2} \mathrm{O}^{+}\right)$which may self modify the medium properties. To study such a possible influence, we have extended our study to the case of electron solvation in ionic solutions.

3.2 ElECtron SOlVATION IN AQUEOUS FERROCYANURE SOLUTION. - The femtosecond ultraviolet photochemistry of aqueous solution of $\mathrm{Fe}\left(\mathrm{CN}_{6}\right)^{4-}(0.45 \mathrm{M})$ permits the process of electron solvation in aqueous concentrated ionic solutions to be investigated. Femtosecond photolysis of an aqueous solution of ferrocyanide ion was performed using $310 \mathrm{~nm}$ pulses of $100 \mathrm{fs}$ duration. At this wavelength, it is expected that the quantum yield of aqueous electron formation is near 0.1 [17]. Previous photochemical studies of the ferrocyanide ion have established that the excited state of $\mathrm{Fe}\left(\mathrm{CN}_{6}\right)^{4-}$ in water may dissociate in a thermally activated process to yield a solvated electron in less than $0.3 \mathrm{ps}$ [5] according to the following equation :

$$
\begin{aligned}
\mathrm{Fe}\left(\mathrm{CN}_{6}\right)^{4-}+\mathrm{h} \mu \rightarrow\left[\mathrm{Fe}\left(\mathrm{CN}_{6}\right)^{4-}\right]^{*} \rightarrow \\
\rightarrow \mathrm{Fe}\left(\mathrm{CN}_{6}\right)^{3-}+\mathrm{e}_{\mathrm{d}}^{-} .
\end{aligned}
$$

This photochemical process competes with a reversible photoaquation reaction leading to the formation of a pentocyanoaquo complex :

$$
\begin{aligned}
{\left[\mathrm{Fe}\left(\mathrm{CN}_{6}\right)^{4-}\right]^{*}+\mathrm{H}_{2} \mathrm{O} } & \rightleftarrows \\
& \rightleftarrows\left[\left(\mathrm{Fe}\left(\mathrm{CN}_{5}\right) \mathrm{H}_{2} \mathrm{O}\right)^{3-}\right]+\mathrm{CN}^{-} .
\end{aligned}
$$

The time-resolved spectroscopy data in the infrared and visible (Fig. 5) demonstrate that photodetachment of electron and subsequent solvation proceed through at least one intermediate state. This intermediate appears with a time constant of $120 \pm 20 \mathrm{fs}$ and relaxes towards a solvated species following a first order kinetics with a time constant of $240 \pm 30 \mathrm{fs}$. At $720 \mathrm{~nm}$, the signal follows the kinetics equation

$$
\begin{aligned}
A_{(t)}^{\prime}=A^{0}(1 & -\left(1 /\left(T_{2}-T_{1}\right)\right) \times \\
& \left.\times\left(T_{2} \exp -\left(t / T_{2}\right)-T_{1} \exp \left(-t / T_{1}\right)\right)\right)
\end{aligned}
$$

as expected after a first-order kinetics of appearance of the precursor of the solvated species with
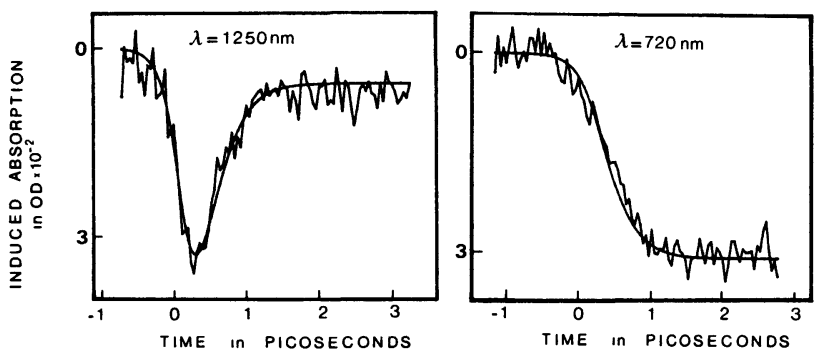

Fig. 5. - Kinetic results obtained at 1250 and $720 \mathrm{~nm}$ after femtosecond UV photolysis of aqueous solution of sodium ferrocyanide $(0.45 \mathrm{M})$. The smooth lines indicate the computed best fits of the experimental curves assuming an appearance time $T_{1}=110 \mathrm{fs}$ for the infrared species and its relaxation $T_{2}=240 \mathrm{fs}$ towards the solvated state.

$T_{1}=120 \pm 20 \mathrm{fs}$ as observed at $1250 \mathrm{~nm}$ and a subsequent relaxation with $T_{2}=240 \pm 30 \mathrm{fs}$.

This behaviour which is identical to what has been observed in pure liquid water demonstrates that electron solvation in concentrated solution of ferrocyanide ion does not proceed through a direct electron capture by pre-existing deep traps as suggested previously [5]. So, the presence of the negative ion $\mathrm{Fe}\left(\mathrm{CN}_{6}\right)^{4-}$ at high concentration does not restructure the immediate water environment and does not provide more favorable spatial distribution of deep trapping sites than in pure liquid water. The reactivity of electron with concentrated ionic aqueous solutions proceeds through at least two transitions involving a transient localized state absorbing in the infrared (Fig. 4) and a subsequent fully solvated state. The time constant that we find for the appearance of the IR absorbing species and its decay toward solvated state are similar in pure water and aqueous ionic solution. This implies that the existence of the presolvated state of electron is neither influenced by the method of electron photoejection nor by the ionic strength of the polar medium in the range $0-0.45 \mathrm{M}$.

Assuming, from previous experiments [18], that thermalization and solvation distance of electron with exogenous energy around $1 \mathrm{eV}$ is about 40 angströms, it can be inferred that many of the quasi free electron with excess kinetics energy escape from the vicinity of the cation $\left(\mathrm{H}_{2} \mathrm{O}^{+}\right)$or the anion $\mathrm{Fe}\left(\mathrm{CN}_{6}\right)^{3-}$ and that long range Coulomb type interactions do not influence neither the initial presolvation nor the configurational relaxation of the medium in the electronic field of the charge. In particular we have no evidence of geminate recombination of presolvated or solvated electrons.

The very first risetime for the appearance of the presolvated state of electron in pure water and aqueous ionic solution implies that the efficient mechanism involved in the localization process does not require large structural reorganization of water molecules in the vicinity of the electron. We may 
speculate that presolvation of excess electron in concentrated ionic solution is promoted, as expected for pure liquid water, by statistical fluctuations in the electronic charge density of the solvent molecules. However the complete understanding of the electron localization process implies knowledge of the distribution and the structure of initial shallow traps. The exact identification and understanding of bound surface states and microscopic trapping sites in concentrated ionic aqueous solutions need more theoretical investigation with tools like path integral techniques or molecular dynamics simulations by Monte-Carlo methods.

In conclusion, the femtosecond photoionization of water and inorganic anion by high power UV pulses induces an injection process of excess electrons in polar medium and furnishes useful information on the basic unresolved question of kinetics and mechanisms of electron localization and solvation in aqueous environment. This paper demonstrates that the electron solvation in pure liquid water and concentrated ionic solution at room temperature proceeds through at least two transient steps with, in particular, an infrared absorbing presolvated state. These data support neither the assumption of the predominant role of the high density of pre-existing deep traps into which the electron fall directly after photoejection nor the existence of a pure dielectric relaxation mechanism for the final energy rearrangement linked to the solvation step. Such data are fundamental for theoretical investigations exploring the obvious quantum mechanical character of the structural and dynamical properties of the electron in polar fluids with great ionic strength.

\section{References}

[1] Hart, E. J., Boag, J. W., J. Am. Chem. Soc. 84 (1962) 4090

[2] Rentzepis, P. M., Jones, R. P., J. Chem. Phys. 59 (1973) 766.

[3] Huppert, D., Struve, W. S., Rentzepis, P. M., J. Chem. Phys. 63 (1975) 1205.

[4] Kenney-Wallace, G. A., Jonah, C. D., J. Phys. Chem. 86 (1982) 2572.

[5] Wiesenfeld, J. M., IpPen, P., Chem. Phys. Lett. 73 (1980) 47.

[6] Wallovist, A., Thirumalai, D., Berne, B. J., J. Chem. Phys. 85 (1986) 1583.

[7] Jonah, C. D., Romero, C., Rahman, A., Chem. Phys. Lett. 123 (1986) 209.

[8] Schnitker, J., Rossky, P. J., J. Chem. Phys. 86 (1987) 3462.

[9] Schnitker, J., Rossky, P. J., Kenney-Wallace, G. A. 85 (1986) 2986.

[10] Migus, A., Antonetti, A., Etchepare, J., Hulin, D., Orszag, A., J. Opt. Soc. Am. B 2 (1985) 584.
[11] Gauduel, Y., Migus, A., Martin, J. L., Lecar PENTIER, Y., ANTONETTI, A., Ber. Bunsen-Ges. Phys. Chem. 89 (1985) 218.

[12] Nikogozyan, D. N., Oraevsky, A. A., Rupasov, V. I., Chem. Phys. 77 (1983) 131.

[13] Gauduel, Y., Migus, A., Martin, J. L., AntoNETTI, A., In Ultrafast Phenomena V, Eds Fleming and Siegman, Springer-Verlag (1986) 427.

[14] Migus, A., Gauduel, Y., Martin, L. J., ANTONETti, A., Phys. Rev. Lett. 58 (1987) 1559.

[15] Fauchet, P. M., Hulin, D., Migus, A., ANTONETti, A., Kolodzey, J. and Wagner, S., Phys. Rev. Lett. 57 (1986) 2438 and references therein.

[16] Calef, D. F., Wolynes, P. G., J. Chem. Phys. 78 (1983) 4145.

[17] Shirom, M., Stein, G., J. Phys. Chem. 55 (1971) 3372.

[18] Krohn, C. E., Antoniewicz, P. R., Thompson, J. C., Surf. Sci. 101 (1980) 241. 